\title{
Type 3 Transformation Zone of the Cervix and Risk of Missed Lesions during Cervical Cancer Screening with Visual Methods: A Case Report from Cameroon
}

\author{
Simon M Manga, PhD ${ }^{1,2^{*}}$, Alan T Tita, MD, PhD ${ }^{2,3}$, Warner $K$ Huh, MD ${ }^{2,4}$, Calvin Ngalla, \\ $B N S^{1}$ and Margaret I Liang, MD, MS $S^{2,4}$
}

${ }^{1}$ Women's Health Program, Cameroon Baptist Convention Health Services, Bamenda, Cameroon

${ }^{2}$ Center for Women's Reproductive Health, Department of Obstetrics and Gynecology, University of Alabama at Birmingham, Alabama, USA

${ }^{3}$ Division of Maternal Fetal Medicine, Department of Obstetrics \& Gynecology, University of Alabama at Birmingham, Alabama, USA

${ }^{4}$ Division of Gynecologic Oncology, Department of Obstetrics \& Gynecology, University of Alabama at Birmingham, Alabama, USA

*Corresponding author: Dr. Simon Manga, Post Doctoral Scholar, Women's Health Program, Cameroon Baptist Convention Health Services, Bamenda, Cameroon; Center for Women's Reproductive Health, Department of Obstetrics and Gynecology, University of Alabama at Birmingham, Alabama, USA, Tel: +237-671-863-768

\begin{abstract}
Type 3 transformation zone (TZ) of the cervix poses a challenge in cervical cancer screening with visual techniques because of the potential for missing important precancerous lesions. This paper presents two case reports of women with Type 3 TZs who were screened with visual methods at the Cameroon Baptist Convention Health Services, had reassuring screening results, but presented soon after with invasive cervical cancer (ICC). It is likely that at the time of their initial screening, they already had high grade precancer or early ICC that were involving the TZ beyond the examiner's view. If there were a technique to reliably convert the Type 3 TZ to Type 1 or 2, a hidden lesion may have been exposed and allowed for earlier diagnosis of a precancer or earlier stage ICC. Without acceptable and affordable techniques to convert Type 3 TZ to Type 1 or 2, women in low-and-middle income countries (LMICs) where visual methods are largely the primary screening method for cervical cancer, may continue to have missed lesions that could have been treated earlier. Thus, it is paramount to identify a technique that can reliably convert Type 3 TZ into Types 1 or 2 in LMICs. Among promising techniques for converting Type 3 TZ to Types 1 or 2, misoprostol appears to be the most feasible in LMICs. It is therefore important to conduct a clinical trial on the use of misoprostol in converting Type 3 TZ into Types 1 or 2 in LMICs.
\end{abstract}

\section{Keywords}

Uterine cervical neoplasia, Developing countries, Cameroon, Precancerous conditions

\section{Introduction}

Type 3 transformation zone (TZ) of the cervix poses a challenge in cervical cancer screening with visual techniques because of the potential for missing important precancerous lesions which can progress into invasive cervical cancer (ICC) prior to detection. The $\mathrm{TZ}$ is the most important portion of the cervix to be examined because most cervical precancers and ICC originate from there [1]. The TZ is the area between the original squamo-columnar junction ( $\mathrm{SCJ}$ ) and the new SCJ. The SCJ is where the squamous and columnar epithelium meet. As women age, the $\mathrm{SCJ}$ is driven into the endocervical canal [2]. When the SCJ is located at the ectocervix and can be visualized at $360^{\circ}$, it is known as Type 1. Whenpart or all of the SCJ is in the endocervical canal but can be visualized at $360^{\circ}$ with/ without manipulation of the speculum, it is known as

Citation: Manga SM, Tita AT, Huh WK, Ngalla C, Liang MI (2021) Type 3 Transformation Zone of the Cervix and Risk of Missed Lesions during Cervical Cancer Screening with Visual Methods: A Case Report from Cameroon. Obstet Gynecol Cases Rev 8:195. doi.org/10.23937/2377-9004/1410195 Accepted: March 20, 2021: Published: March 22, 2021

Copyright: (C) 2021 Manga SM, et al. This is an open-access article distributed under the terms of the Creative Commons Attribution License, which permits unrestricted use, distribution, and reproduction in any medium, provided the original author and source are credited. 
Type 2. When part or all of the SCJ is at the endocervical canal and cannot be visualized at $360^{\circ}$, it is known as Type 3 [3]. In this paper, the term SCJ is used to denote the new SCJ.

Cervical cancer visual screening methods involve visual inspection of the cervix with acetic acid (VIA) and Lugol's iodine (VILI) [4]. These methods are easy to perform, cheap, and do not require sophisticated equipment. The world health organization (WHO) recommends VIA as a primary screening test for cervical cancer in low-and-middle income countries (LMICS) [1]. The visual screening methods can be further enhanced with magnifying equipment such as colposcopy or digital cervicography (DC). It has been shown that women with Type 3 TZs undergoing regular cervical cancer screening with visual methods have a four-to five-fold increased risk of developing ICC than women who undergo regular screening with non-visual methods such and cytology [5]. In the case of a Type 3 TZ with negative visual screening result, the woman returns home with a reassurance; however, it is possible she has a preinvasive lesion that was not visualized.

In high-income countries, women with Type 3 TZs undergoing cervical cancer screening usually undergo endocervical curettage to sample the portion of the TZ that has been driven into the endocervical canal [6]. In LMICs, obtaining pathological results from endocervical sample is challenging because pathologists lack the appropriate equipment. With the emergence of human papilloma virus (HPV) DNA testing, the screening paradigm will eventually shift from identification of cervical precancer to oncogenic HPV. However, those positive for oncogenic HPV will still require VIA/VILI to rule-out pre-invasive or invasive lesions meaning visual screening methods will not be obsolete anytime soon. Thus, a feasible solution for women with Type $3 \mathrm{TZ}$ is needed in LMICs.

The Cameroon Baptist Convention Health Services, a large faith-based health care organization in Cameroon runs the largest cervical cancer screening program in Cameroon called the women's health program (WHP). The program which relies principally on VIA/VILI-DC has been described in detail in our previous work [7]. In WHP, women who screened negative irrespective of the type of TZ are given a three-year follow-up appointment if they are HIV negative and one-year follow-up appointment if HIV positive.

Below are two case studies of women who were screened with VIA/VILI-DC within WHP with Type 3 TZs and were given reassuring results but developed ICC shortly thereafter.

\section{Case One}

This is a case of a 46-year-old G4P4004 HIV negative woman who was married and a farmer by profession. She first underwent VIA/VILI-DC in August 2018 at WHP

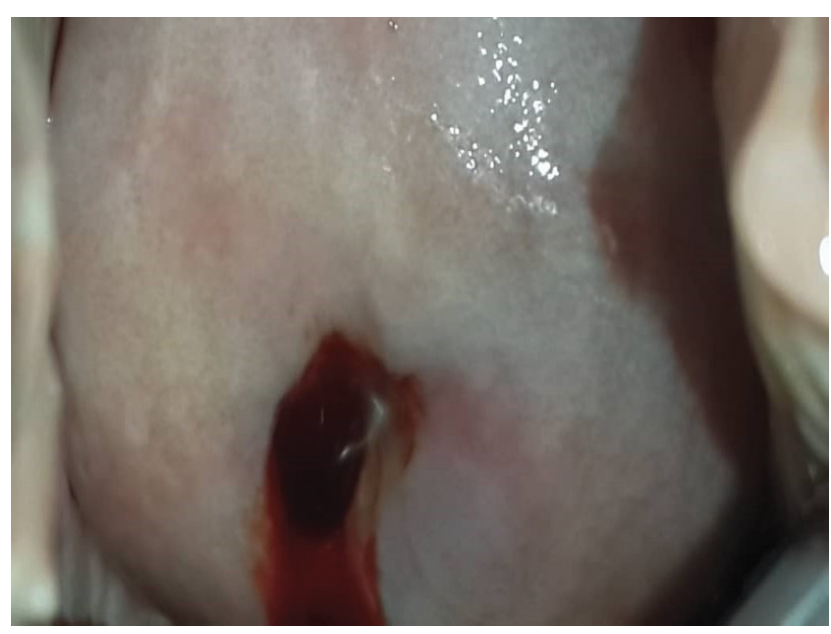

Figure 1a: Visual inspection with acetic acid of Type 3 transformation zone of Case One.

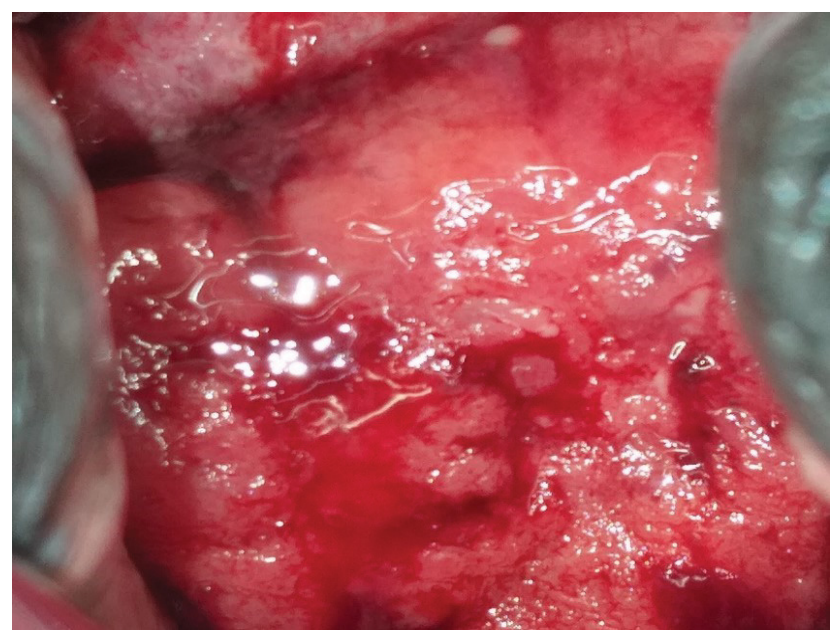

Figure 1b: Invasive cervical cancer of Case One.

at Mbingo Baptist Hospital (MBH). The woman had a Type 3 TZ and no visible lesion on VIA-DC (Figure 1a). She was given a follow-up appointment for three years.

In June 2019, the woman consulted at the outpatient department at $\mathrm{MBH}$ with history of profuse, persistent, malodorous vaginal discharge for two weeks. She had been treated elsewhere with anti-bacterial agents including amoxicillin and metronidazole without improvement. During this visit, a vaginal culture and sensitivity of a vaginal smear with sensitivities was done and Candida albicans and Streptococcus faecalis were isolated, which were sensitive to ciprofloxacin and fluconazole, respectively. She was treated with fluconazole $200 \mathrm{mg}$ single dose orally and ciprofloxacin $500 \mathrm{mg}$ twice daily for 10 days without improvement.

In April 2020, she returned to the WHP clinic at $\mathrm{MBH}$ with history of vaginal bleeding for two months. Upon speculum examination, she was found to have a broad, exophytic cervical mass that was friable (Figure 1b). The lesion extended to the distal lower third of the vaginal wall. On recto-vaginal exam, there was bilateral parametrial involvement. Pelvic ultrasound 


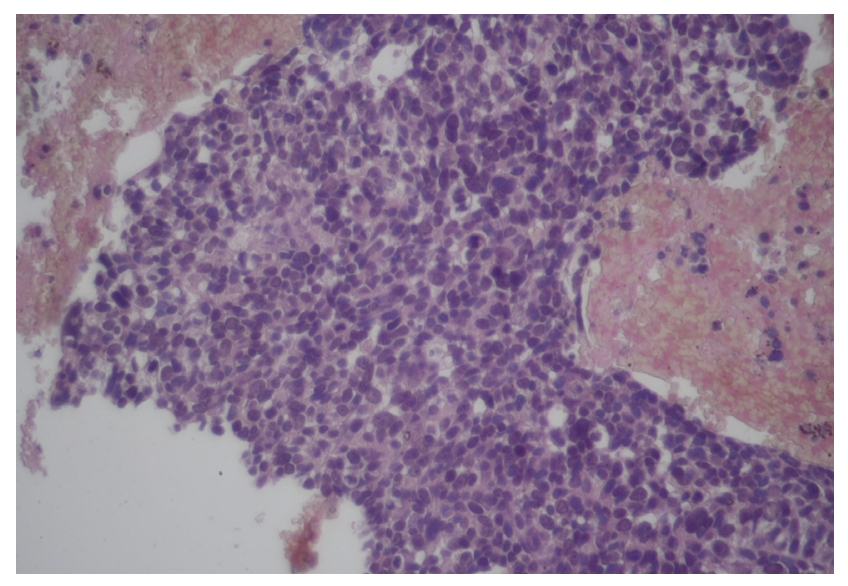

Figure 1c: Histological appearance of the cervical cancer of Case One

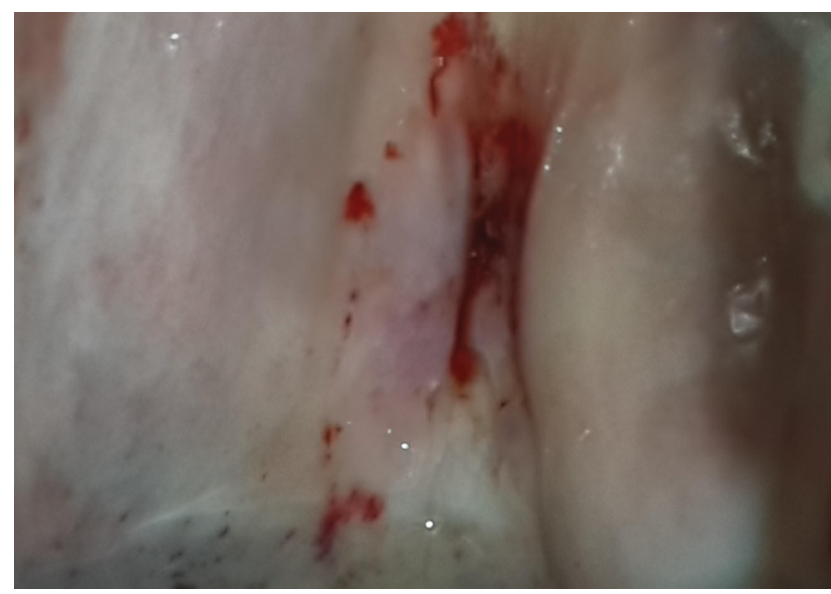

Figure 2a: Visual inspection with acetic acid of Type 3 transformation zone of Case Two.

demonstrated a cervical mass and right hydronephrosis. The clinical impression was a 2014 International Federation of Gynaecology and Obstetrics (FIGO) stage IIIB ICC. An ectocervical punch biopsy was performed. The final pathology result confirmed an invasive squamous cell carcinoma of the cervix (Figure 1c). She was then referred for palliative radiotherapy at Douala General Hospital.

\section{Case Two}

This is the case of a 70-year-old G7P7003 HIV positive widow who had been on antiretroviral, Tenolam, for less than a year before her first cervical cancer screening. Upon initiation of her HIV treatment, her CD4 count was 606 cells/ $\mu$ land CD4\% 22.5\%. She was otherwise healthy with no additional medical conditions. Her first VIA/VILIDC was performed in October 2018 at the WHP clinic at $\mathrm{MBH}$. She had a Type $3 \mathrm{TZ}$ with no identifiable lesion, so she was reassured and asked to return in one year for follow-up screening given her HIV status (Figure 2a). She missed her follow-up appointment scheduled for October 2019. She later returned to the WHP clinic at $\mathrm{MBH}$ in August 2020 with history of intermittent vaginal spotting for one month. Speculum exam revealed an

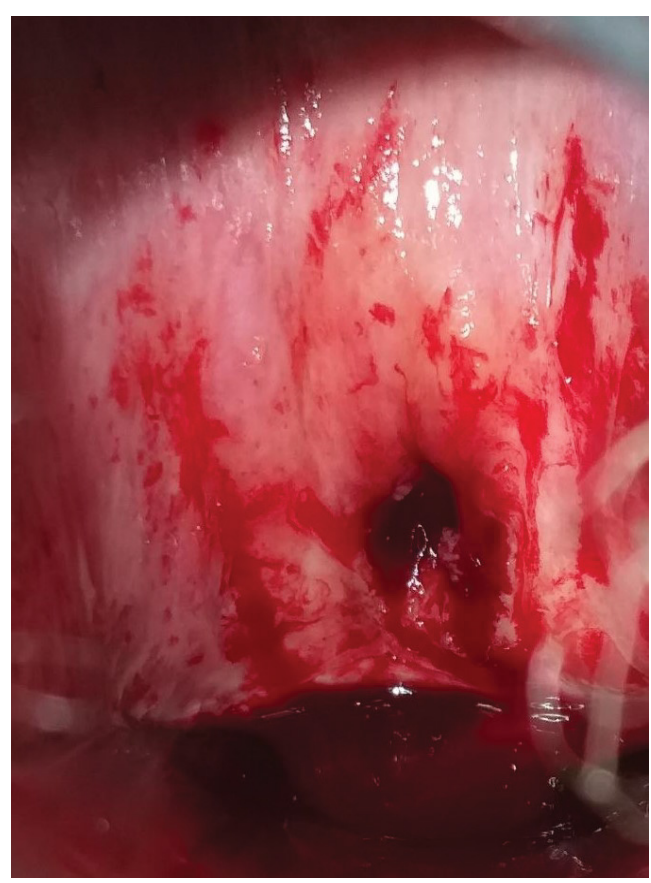

Figure 2b: Invasive cervical cancer of Case Two.

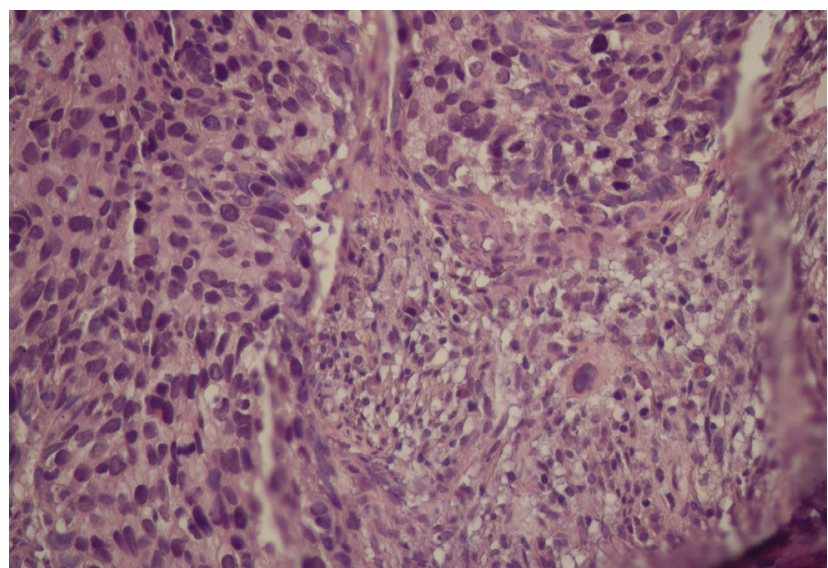

Figure 2c: Histological appearance of the cervical cancer of Case Two

inflamed cervix with mild erosion. The VIA-DC was uncertain because it was between severe cervicitis and early ICC (Figure $2 \mathrm{~b}$ ). An ectocervical punch biopsy was performed and the histopathology report confirmed an ICC (Figure 2c). Pelvic ultrasound and chest X-ray were normal. The cancer was 2014 FIGO stage IB1 and she was referred for a radical hysterectomy.

\section{Discussion}

In the majority of cases, cervical cancer begins with a precancerous lesion due to one or more types of oncogenic HPV, which takes an average of 10 years to become an ICC [1]. The women in this case study both developed ICC in less than three years after receiving reassuring screening results on VIA/VILI-DC. Given the Type $3 \mathrm{TZs}$, it is likely that at the time of their initial screening, they already had high grade precancer or early ICCS that were involving the TZ beyond the 
examiner's view. If there were a technique to reliably convert the Type 3 TZ to Type 1 or 2, a hidden lesion may have been exposed and allowed for earlier diagnosis of a precancer or earlier stage ICC. Treatment of cervical precancer is effective at preventing progression to ICC and treatment of early ICC is associated with higher survival rates $[1,6]$. Without acceptable and affordable techniques to convert Type 3 TZ to Type 1 or 2, women in regions where visual methods are the primary screening method for cervical cancer who have Type 3 TZ may continue to have missed lesions that could have been treated earlier.

Two pharmacological regimens have been evaluated in converting Type 3 TZs to Type 1 or 2 including intravaginal misoprostol and intravaginal or oral estradiol $[7,8]$. However, there is not enough evidence to support implementation of these regimens in clinical practice because the few randomized control trials (RCTs) that have been conducted had small sample sizes and were inadequately powered. Moreover, none of these RCTs were conducted in Africa. Thus, it would be clinically important to conduct an RCT with adequate sample size in Africa, where VIA is widely used as primary screening test and where the burden of cervical cancer is high [1]. Misoprostol has multiple potential advantages. Vaginal and oral estradiol are administered in multiple doses, while misoprostol is administered in single dose $[7,8]$. Also, misoprostol is included in the essential drug list, which makes it widely available and affordable in sub-Saharan Africa compared to estradiol [9]. In addition, the single dose regimen of misoprostol favors the "see-and-treat" approach for cervical cancer prevention recommended by WHO for LMICs.

A shorter follow-up interval for re-screening may have been helpful to these women as the lesion might advance rapidly beyond the hidden portion of the $T Z$ towards the ectocervix. Given the natural history of cervical cancer, it is surprising that case one manifested signs and symptoms of ICC less than one year after her initial screening particularly considering her HIV negative status. Unfortunately, type of TZ has not been taken into consideration when designing guidelines for cervical cancer screening and re-screening. Case two missed her one-year follow-up appointment and manifested signs and symptoms of ICC shortly thereafter. Cervical cancer prevention programs whose primary screening methods are VIA/VILI could consider shorter follow-up for women with Type 3 TZs. Unfortunately, follow-up is not optimal in LMICs even among women diagnosed with precancers [10]. Therefore, following up women with Type 3TZ who have reassuring results could be even more challenging.

\section{Conclusion}

Type $3 \mathrm{TZ}$ is associated with the risk of missed cervical lesions in programs that rely on cervical cancer screening with visual methods. The risk could potentially be reduced by considering shorter follow-up for women with Type $3 \mathrm{TZ}$, but there are multiple barriers to ensuring reliable follow-up for such women. An acceptable, affordable, and feasible method to convert Type 3 TZ into Type 1 or 2 in LMICs might be a better solution to this problem especially a method that would support the "see-and-treat" approach. Intravaginal misoprostol appears to be a promising solution to this problem. Thus, an RCT with adequate sample size should be conducted in LMICs especially SSA to evaluate the effectiveness of misoprostol in converting Type $3 \mathrm{TZ}$ into Types 1 or 2.

\section{Authors Declaration}

All authors have contributed equally to the work.

\section{Funding}

Dr. Tita was supported for this work by NIH/NCATS grant \#UL1TR003096 (PI: Kimberly) Center for Clinical and Translational Science (Linked award: UL1TR003096, TL1TR003106, KL2TR003097).

\section{References}

1. WHO (2014) Comprehensive cervical cancer control.

2. O'Connor D (2014) Anatomy and histology of normal female lower genital tract. In: Mayeaux EJ, Cox JT, Modern Colposcopy. ( $3^{\text {rd }}$ edn), Wolters Kluwer/Lippincott Williams \& Wilkins Health, New York.

3. Luyten A, Buttmann-Schweiger N, Hagemann I, Sarah S, Gerd B, et al. (2015) Utility and reproducibility of the international federation for cervical pathology and colposcopy classification of transformation zones in daily practice: A multicenter study of the german colposcopy network. J Low Genit Tract Dis 19: 185-188.

4. Ferris DG, Cox JT, Mayeaux EJ (2014) Colposcopy of cervical intraepithelial neoplasia. In: Mayeaux EJ, Cox JT, Modern Colposcopy. ( $3^{\text {rd }}$ edn), Wolters Kluwer/Lippincott Williams \& Wilkins Health, Philadelphia.

5. Q W Cao, Z X You, X Y Qian, D Heng, M Y Tang (2019) Discussion on the diagnosis and treatment of high-grade squamous intraepithelial lesions in post-menopausal women.Chin J Obstet Gynecol 54: 393-398.

6. Cox JT, Chelmow DP, Moscicki AB (2014) Management of abnormal cervical cancer screening. In: Mayeaux EJ, Cox JT, Modern Colposcopy. ( $3^{\text {rd }}$ edn), Philadelphia.

7. Pergialiotis V, Vlachos D-E, Pitsouni E, Perrea D, Vlachos GD (2015) Vaginal misoprostol for overcoming inadequate colposcopies. J Low Genit Tract Dis 19: 257-261.

8. Prendiville WJ, Davis WAR, Davis JO, Sherperd AM (2020) Medical dilatation of the non-pregnant cervix: The effect of ethinyl oestradiol on the visibility of the transformation zone. Br J Obstet Gynaecol 93: 508-511.

9. OMS (2019) World health organization model list of essential medicines. Ment Holist Heal Some Int Perspect.

10. Manga SM, Shi L, Welty TK, Demarco RF, Aronowitz T (2020) Factors associated with treatment uptake among women with acetic acid/lugol's iodine positive lesions of the cervix in Cameroon. Int J Womens Health 12: 495-504. 